\title{
Beyond Misconceptions: Neurobiological and Genetic Associations in Eating Disorders
}

By Sara E. Gorman and Jack M. Gorman, MD

The category "eating disorders" is and has always been a troublesome entity. The disorders within this grouping are incredibly varied, making any kind of thorough understanding of eating disorders extraordinarily trying. In most cases, the general public views anorexia nervosa (AN) and bulimia nervosa (BN) as characterized by opposite behaviors. After all, $\mathrm{AN}$ involves starvation, whereas $\mathrm{BN}$ involves binge eating, clearly the opposite if one considers only caloric intake. Less apparently, people often view the personality types coinciding with each disease to be opposite. BN patients may be characterized as trouble-making, promiscuous, and rebellious, whereas AN patients have always been viewed as perfectionists and obedient.

These kinds of categorical discriminations among the eating disorders may be valid in many cases, but people are more often than not misled in believing that these opposite personalities define and perhaps even cause the illnesses. The danger in invoking these "absolutes" when considering mental illnesses is that people come to associate illnesses with individuals. In believing that quiet perfectionism necessarily coincides with AN, people come to attribute a sickness to a personality type. People even invent social causes for these illnesses, often blaming social pressure to be thin or parental insistence on high achievement. In fact, in a letter to the New York Times dated May 30, 2004, ${ }^{1}$ a writer claims "Some of the currently common syndromes among adolescents-eating disorders, cutting and possibly bipolar disorder-seem to have originated among more affluent kids." Later, the writer suggests, "High-achieving and often stressedout parents might be part of the explanation." In no other medical field would a layperson suggest the cause of an illness with no statistical data or expertise of any kind. Unfortunately, many of these kinds of claims do exist, and they often dilute the severity and reality of the eating disorders as illnesses, casting them as cultural or personal phenomena.

It may, in fact, be true that eating disorders and specific personalities, income, and social pressures do coincide, but simplifying the causes of these complex illnesses to stress and high achievement is clearly insufficient.

The research in this issue of CNS Spectrums shows neurobiological links among the eating disorders $\mathrm{AN}$ and $\mathrm{BN}$, binding them together not in exact causes but in the general recognition of their genetic, hormonal, and molecular bases. Clearly, this kind of research is essential in treating patients. On a different level, this research is the necessary delineation of the biological bases of eating disorders, an incredibly useful tool in showing that there is no one cultural aesthetic or societal system of demands that causes disordered eating. This type of statement can only help in pushing people away from the too-simple conclusion that $\mathrm{AN}$ and $\mathrm{BN}$ begin with a disordered personality and come to the fore with the pressures of disordered families and societies. CNS

\section{REFERENCE}

1. Ehrenreich B. Not a squishy concept. New York Times. May 30, 2004; sect 7:4. 